\title{
El problema de la investigación en la bibliotecología norteamericana: una revisión (1930 - 1960)
}

\section{Susana Sander}

\section{RESUMEN}

En este trabajoin tentamos revisartrein ta años deliteraturabibliotecoló gi canorteamericana con la finalidad de en tre sacaral gunos de los con tex tos en don de se ha plan tea do el pro ble ma de la in ves ti ga ción en esta dis ci pli na, los al can ces y lími tes de su so lución.

\section{ABSTRACT}

In this ar tic le we at tempt to re view thirty years of North Ame ri can li brary scien ce li te ra tu re with the goal of se lec ting some of the contexts where the problem of research in this field of study has been formulated.Certain achie ve ments and li mitations will be noted.

Re sul ta va lio so que en esta épo ca, con una cla ra ten den cia al prag ma tis mo y a la fun ciona li dad del po der, en con tre mos una dis ci pli na que con ti núe la bús que da de una com pren sión cada vez más profunda de su propio actuar. Desde hace más de un siglo, los bibliotecólogos han venido recla mando, pordistintas vías, el es cla re cimien to del carác ter cien tífic co y fil lo sófico de su profesión, poniendo de manifiesto que su vinculación vo ca cio nal con los li bros - no ble y pro fun da ex pre sión de la culturahumana-hasido de gransignificación for mativa. No obstante, ex plicitar lacien tificidadeín do le filosófica de labi bliotecología es enfrentarse a una problemática sumamente compleja constituida, principalmente, por las siguientes preguntas: ¿Qué es en sí misma la bibliotecología? ¿Cuáles son las teorías que la definen y los mé to dos con los que in ves tiga sus obje tos? ¿Cuáles son sus fi nes so ciales? ¿Quélu gar ocu pa dentro de la estructura general de las ciencias?

Uno de los contextos en donde se han planteado algunos temas de esta problemática y donde se han expuesto posibles solu cio nes, ha sido en el de la bi blio te co lo gía nor te ame ri ca na des de prin ci pios de si glo has ta nues tros días. Una sus cin ta revisión de su literatura, nos permitirá entresacar el problema de la investigación y hacer algunas consideraciones críticas, pues nos percatamos que un estudio profundo sobre este tema, rebasa las intenciones de este trabajo.

En las primerasdécadas del siglo XX, des pués de la pu bli cación del es que ma de cla si fi ca ción de Dewey y la fun da ción de la primeraes cuela de Bibliote conomía, so bre salien tes bibliotecarios nor te america noscomien zan ain sistirenla necesidad de integrarprogramas de investigación en las escuelas de biblioteconomía, así como extender las actividades de investigación hacia la práctica bibliotecaria.

Los argumentos en favor de la investigación que proponían los bibliotecarios presentaban distintos niveles:
1. Los re fe ri dos a la po si bi li dad de tras cen der el ca rác ter práctico de la actividad bibliotecaria. ${ }^{(1)}$

2. Los que con si de raban fac tible de sa rrollarun "es píritu científico" sobre la naturaleza empírica que poseían los bibliotecarios. $^{(2)}$

3. Aquellos argu mentos di rigi dosa: sobrevalorarlas ac tividades de investigación, al dotaralbibliotecario deconocimientos que le per mi tie ran te ner una cla ra con cien cia de la fun ción social y educativa que habíaadquirido la biblioteca; manejar con ma yor efi ca cia el ob je to de su ac ti vi dad; y ob te ner ba ses sólidas para responsabilizarse de las necesidades de evaluación y proyección de los servicios bibliotecarios.

Las implicaciones antropológicas y gnoseológicas que estaban en la base de estos argumentos no fueron esclarecidasen esa época aunque ya habían sido abordadas como temas de profunda reflexión por la historia del pensamiento humano des de ha cía casi 20 si glos atrás. Es tas im pli ca cio nes es tán referidas, particularmente, a factores teóricos-cognoscitivos que in ci den y se ge ne ran en las ac ti vi da des prác ti cas las cua les po ten cian la ca pa ci dad em pírica (sen si ble) de los se res humanos, condición de posibilidad necesaria para alcanzar la síntesis del conocimiento teó ri co. Es tas re fle xio nes no se llevaron a cabo, quizás en razón del contexto socio-político y eco nó mi co en que se plan teó el proble ma de la in ves ti ga ción; quizás, también, a causa delos fun da men tos ideoló gi co-filosóficos-empiristas que estaban a la base del proyecto liberal que extendió la biblioteca pública. Esto provocó, en cierto sentido, toda una corrien te de des va lori za ción del tra bajo biblio te ca rio que tuvo, y ha te ni do, una im por tan te fun ción político-e co nó mi ca al dar ele men tos (comola au sen cia de reflexión teórica) que justificaron la anulación o reducción de presupuestoparaactividades deinvestigaciónbibliotecológica y descalificaron, a priori, las capacidades teóricas de los 
bibliotecarios, a la vez que, paradójicamente, sus servicios eran cada vez más necesarios y solicitados.

El tercer grupo de argumentos es uno de los factores que expli ca la de mora en la in ves ti ga ción de la es truc tu ra teó ri ca de la bibliotecología. Estructura teórica reclamada implícitamente en la demanda de investigación. Demora debida a que en es tos ar gu men tos está in mer sa una con cep ción de la in vesti ga ción como ins tru men to prác ti co y no teóri co, como me dio y no como finalidad de desarrollo humano y profesional de losbibliotecónomos y bibliotecarios, en todas las áreas de su actividad práctico- cognoscitiva. En esta tercera argumentación, la investigación no tendría como objetivo transformar una actividad práctica, como la bibliotecaria, en otra con características esencialmente teóricas; por el contrario, la meta que se pre ten de es in tro du cir, a tra vés de la in ves ti ga ción, los me dios que le per mi tan al bi blio te ca rio te ner éxi to en sus funciones, puesto que al investigar obtendría una conciencia lúcida del papel social y educativo de la biblioteca para potenciar los servicios bibliotecarios.

Lain ves ti ga ción ven dría a re sol ver los proble mas téc ni cosinmediatos que el quehacer del bibliotecario enfrenta, al darle un saber más amplio que aquél que surge directamente de su ac ti vi dad y vaacu mu lán do se y cons ti tu yen do su ex periencia.

Como vemos, a diferencia del primer nivel de argumentos donde la fun ción de la in ves ti ga ción es tras cen der el ca rác ter práctico del bibliotecario, en este tercer nivel, la investigación no tiene como finalidad potenciar la función social y educativa de la biblioteca, menos aún, fortalecer la reflexión teórica para que el bibliotecario pueda responder al ¿qué es? ¿para qué es? ¿por qué es? su actividad. Las condicioneshistóricas, y el positivismo, como corriente de pensamiento dominante, determinan que la reflexión bibliotecológica en el ámbito norteamericano no responda, directamente, a necesidades teóricas, sino a demandas prácticas.

Ve mos, en es tas pri me ras dé ca das del Si glo XX, como la tendencia de formar"buenos"administradores de bibliotecas se ini cia ba. Por ello, se bus ca ba que el sa ber, ob te ni do por la investigación y no sólo el producido por la experiencia, fuera más apto para lograr la eficacia práctica.

Lo anterior, permite comprender por qué la reflexión bibliotecológica, de principios de siglo en Estados Unidos de Norteamérica, argumenta "teóricamente" la necesidad de incorporar actividades de investigación en el quehacer bibliotecario, sin desarrollar previamente una teoría que explique, tan to los fe nó me nos bi blio te ca rios como los bi blio teco nó mi cos a tra vés de un con jun to de le yes que fun da men ten, tam bién, los mé to dos y téc ni cas de in ves ti ga ción para di chos fenómenos, desarrollo necesario de una teoría que fue planteado en esa década, sin tener eco. ${ }^{(4)}$

La ausencia de reflexión bibliotecológica en ese sentido promovió que, en las siguientes décadas, se presentara un conjun to de pro pues tas pararea li zar in ves ti ga ción con ellla ma do "modelo de investigación científica" que se reducía, en su mayor parte, a un método cuantitativo siguiendo, en cierto sentido, la propuesta hecha por Whitworth. ${ }^{(5)}$

Así, una constante en los escritores norteamericanos de esa época es la apelación a métodos científicos probados con éxito en otros campos, que fomentan la creencia de que es posible transformar una actividad práctica en ciencia con la sola incorporación del método cuantitativo o sus técnicas; identificando ciencia con investigación e investigación con estadística. Medios, todos, subordinados alobjeti vobuscado: la eficacia.

En congruencia con lo anterior, la indagación en otras naciones sobre la existencia de reflexiones bibliotecológicas o la propia producción de teorías bibliotecológicas en Norteamérica, no era prioritaria. Se trataba de resultados no de comprensiones.

Sin em bar go, hay que res ca tar en es tas con cep cio nes so bre el papel de la investigación, la idea fundamental de que la $\mathrm{Bi}$ blio te cología es una ac ti vi dad reflexi va y sis te mática que, por lo mis mo, re cu rre a ins tru men tos me tó di cos para re sol ver los problemas que enfrenta y no ape la a la fan ta sía, a la ima gi nación o a los buenos propósitos.

Ante la au sen cia de un es cla re ci mien to cien tífico y filo sófico de la actividad bibliotecológica, proliferaron las discusiones para de ter mi nar cuáles eran los mé to dos de in ves ti ga ción que debían utilizarse y cuáles no; culminando esta polémica en los años se sen ta con la Con fe ren cia so bre mé to dos de in ves tigación en Bibliotecología llevada a cabo en Illinois, Estados Unidos de Norteamérica. ${ }^{(6)}$

Sin embargo, el problema de establecer los métodos que debían in cor porar se a la in ves ti ga ción pare cía plan tear se ex ternamentealdesarrollohis tórico delaactividadbiblio te cológi ca y era indiferente a la existencia de bibliotecas cuyas colecciones, des de la antigüedad, requirieron un conjunto de actos reflexivos y organizados conscientemente — como la catalogación - para su preservación, uso y transmisión. Actos todos, con una racionalidad susceptible de ser abstraída, conceptualizada y generalizadacomocamino de acción, método y factor teórico.

Si bien en los años se sen ta hubo dis cu sio nes so bre la elec ción de mé to dos ex ternos a labiblio te cología, parain cor porarlos a ella, se dio también una continua fructificación de las actividades de clasificación y catalogación; actividades que desde épocas an ti guas los bi blio te ca rios ha bían ve ni do de sa rrollando, influenciados por los conocimientos y sa be res de su época, y que se expresaban en esta década, como la amplia producción de investigaciones sobre principios, leyes y reglas para la ela bo ra ción de thesau rus, ${ }^{(7)}$ la crea ción de ca te go rías y conceptos para indizados automáticos, ${ }^{(8)}$ etc.

Resulta evidente que, ante la ausencia de una comprensión teórica de labiblio te cología, la dis cu sión sobre mé to dos fue ra tan polémica; se trataba de la eficacia. Por ello, no era relevante la procedencia de dichos métodos, ni su adecuación o 
no a los fe nó me nos que ha brían de abor dar, sino cuá les mé todos habían sido probados con éxito en otras disciplinas.

Es cla ro que, el ob je ti vo que se pre ten de, re quie re de téc nicas adecuadas para realizarse, sin embargo, hay que advertirque los mé to dos, en el caso de la cien cia, no se eli gen por los fi nes que se pre ten den, sino por la per ti nen cia de és tos con los fe nómenos que se han de explicar. Sin la adecuación entre métodos y fenómenos, el fin explicativo no se alcanza.

Ve mos por lo tan to que, al me nos en los pri me ros trein ta años delabibliotecologíanorteamericanaenfocadaal problemade la investigación, se vivían unas condiciones históricas de tal mag ni tud que le im pe dían no sólo bus car so lu cio nes en otros países, pues la guerra del 39 alteraba todo diálogo, sino también la im posi bi li taban parahaceruna re cu peración del pasadobibliotecológico.Pasado cuya rique za de ele men tos teó ricos sigue siendo potencial para la comprensión y dinamización de la bibliotecología. Pasado, pero también bas topresentebibliotecológicoque podría habersidorecuperado en los sesenta.

Todasesas reflexiones meto dológicas y epis te mológicas, hubieranpermitido a los preocupados por las cuestiones de métodos para la bibliotecología, la paulatina pero segura construc ción de su es truc tu ra teó ri ca como lo de man dó Shaffer en $1968 ;{ }^{(9)}$ ya que el ma te rial teó ri co es ta ba pre sen te en esa con tinua producción de principios, leyes, métodos, técnicas y reglas de clasificación, catalogación, restauración, administración, planeación y construcción de bibliotecas, etc.; y dentro de las concepciones que formalizaron la institucionalización de la profesión bibliotecológica desde finales del siglo XIX, en Ale ma nia, has ta nues tros días; ma te rial que era lo su ficiente men terico y profun do para la ela bora ción de di chaes truc tura.

Además de la ausencia de problemas claves para la elaboración del cuerpo teórico de la dis ci pli na, otra con se cuen cia de la in tro duc ción de mé to dos aje nos a ella, para rea li zar ac ti vidades de investigación, es la violencia que tienden a ejercer so bre la mis ma, al im po ner le una for ma o ca mi no que se debe se guir para lle var a cabo la ac ti vi dad; for ma o ca mi no abs traído de otras dis ci pli nas; sin per ca tar se de que, los con te ni dos y procedimientos producidos por el desarrollo histórico de la biblitotecología, tenían ya una forma e implicaban un conjunto de métodos y técnicas que era sólo necesario estructurar, interrelacionar, conceptualizar y categorizar.

En suma, es po si ble afir mar que, para la dé ca da de los años sesenta, existía ya un conjun to de conocimientos, saberes, actitu des y proce di mien tos en elám bi to de labiblio te co logía, que re que rían de una se rie de pre gun tas y plan tea mien tos cien tíficos y filosóficos para que, en su solución, se conformara explícitamente un cuerpo teórico que sirviera de base para fundamentar los métodos y técnicas que se habían venido utilizando y, en su caso, derivar creativamente aquellos que fuesen necesarios.

Para finales de la década del sesenta, en la literatura norte- americana, que busca reflexionar sobre la actividad bibliotecológica, en con tramosuna variacióncualita ti vaenelcarácter de la críti ca a ese ám bi to, críti ca que co mien za a de tec tar proble mas cla ves en su in te rior. Así, de la críti ca a lo que se lla mó "La naturaleza empírica de los bibliotecarios",o au senciade un espíritu científico que se crearía con la incorporación de actividades de investigación, se pasa a la crítica de la naturaleza de la investigación misma, al determinársele como: débil, fragmentaria y orientada a problemas inmediatos. ${ }^{(10)} \mathrm{Ca}$ rácter negativo, se dice, por cuanto la reduce a producir estudios puramente descriptivos en donde está ausente una re fle xión so bre su ra zón de ser o so bre las cau sas de los fe nómenos que dichos estudios describen.

Lain ves ti ga ción débil, frag men ta ria y sin ca rác ter acu mu la tivo te nía su cau sa, se gún es tos pen sa do res, en lo que se lla mó: fal ta de un cuer po teóri co de la dis ci pli na que per mi tie ra la integración, el fortalecimiento y la continuidad de los conocimientos produ ci dos por la in ves ti ga ción y, con ello, la vin cur lación de las distintas especialidades que se han ido con for man do a tra vés de la his to ria de la pro pia in ves ti ga ción disciplinaria.

Resultaintere san te observarque, aun que se ge ne ra lizaloque podríamos llamar "conciencia de la falta" de una teoría, no au men ta el nú me ro de in ves ti ga do res que cu brantal au sen cia o de investigaciones para elaborar la teoría. Sí se continúa, em pero, re pi tien dolas ac ti tu des asu mi das en los años trein ta, cuandoserelacionabaintrínsecamenteinvestigación con eficacia, donde la efi ca cia era la res pues ta a la pre gun ta so bre la función queten dríalain vestigación paraladisciplina, el para quesirvelain vestiga ción. Ahora van a prolife rarestu dioscuyos ar gu men tos se re fe ri rán a la fun ción de la teo ría en la disciplina, el para qué sirve la teoría. Función que se dirige a dos ámbitos fundamentalmente; para probar un conjunto de hipótesis a través de la investigación experimental (Goldstein, 1968) ${ }^{(11)}$ y para dotar de status a la profesiónbibliotecológica, demandado por la madurez que ella ha alcanzado (Shaffer, 1968). ${ }^{(12)}$

Elcon tex to de los años se sen ta nos per mi te com pren der, tan to las razones ex ter nas de la de man da de una teo ría bi blio te co lógi ca, como aque llas que han im pe di do su rea li za ción ex plícita. En esta época, ya se ha bía ge ne ra li za do, en dis tin tos ni veles de la sociedad, la llamada revolución tecnológica que se de sen ca de nó en la dé ca da del 40 , cuan do las po ten cias de entonces promovieron la necesidad de un nuevo reparto del mun doy de sarrollaronellla ma do "Proyecto Manhattan", que con jun tó a dis tin tos hom bres de cien cia para la pro duc ción de la bomba atómica.

Los subproductos de las investigaciones nucleares, después de ha ber pro ba do su efi ca cia en elárea mi li tar, se ge ne ra li zaron hacia otros campos, principalmente con la innovación tecnológica de la informática la cual, aplicada al sector productivo, trajo consigo la automatización.

La au to ma ti za ción se vino a con ver tir en la esen cia de la re volu ción tec no ló gi ca, per mi tien do que lle ga ra a su pun to cul mi- 
nan te la ló gi ca del de sarrollo ca pita lis ta fun da da en el proce so devaloriza ción delca pi tal.Porello, la re volu ción tec nológica se pre sen tó como in tro duc ción, en to dos los ám bi tos de la sociedad y no solamente el productivo, del principio automático, de un conjunto de innovaciones en los procesos que hacían ma ni fies to que co men za ba a do mi nar la apli ca ción de un saberabs trac to y formal. Proce sos productivos, económicos y sociales, fun da dos cada vez más profun da men te so bre la base de ecuaciones y algoritmos: sobre la cuantificación.

La nue va tec no lo gía se ge ne ra con los mi cro cir cui tos o circuitos in te gra dos, re sul ta do del gran de sa rrollo que al can za laindustria de los semiconductores o conductores eléctricos, logran do una gran pro yec ción al in te grar se a la in dus tria de las computadoras. Integración de la cual surgirálainformáticaa la que se le des cri be como: cien cia del proce sa mien to ló gi co y automático de la información, que comprende el estudio de los me dios, mé to dos y fun cio na mien to del procesa mien to de la información y de sus campos de aplicación.

Si bien los microcircuitos fueron des cu bier tos en 1959, es un hecho que para los sesenta, la supuesta vinculación de la investigación científica y las innovaciones tecnológicas se habían generaliza do, trayen docon sigolarevolu cióncuali tativa de la cien cia como una ac ti vi dad que, ade más de ex pli car simplemente las leyes y fenómenos de la naturaleza, provocaba, al apli car se a la in dus tria a tra vés de las com pu ta do ras, ace leradasinnovaciones tec noló gi cas que permitían la profun dización y dinamismo del capitalismo.

En este con tex to su ma men te con fu so con res pec to a la ve racidad de la conversión de la ciencia en factor de producción e innovacióntecnológica, la demandadecientificidad de muchas ac ti vi da des so cia les, con el ob je ti vo de ace le rar las in novaciones tecnológicas y aumentar intensivamente el capital, comen zóa ge ne ra li zar se como una fun ciónideoló gi carequerida para la producción de conocimientos aptos para nuevos cambiostec nológicos. Sin embargo, laimposibilidadeincostea bi li dad de ace le rar las trans for ma ciones en las dis tin tas actividades, particularmente técnicas, para que alcanzaran el "sta tus" cien tífico, trajo con si gola prolife ración de lain ves tigación aplicada vinculada directamente a las empresas y generada por equipos de investigación — como en el proyecto Manhattan - interdisciplinarios, para resolver de manera direc ta el proble ma de la pro duc ti vi dad que im pe día al can zar el éxito en la competencia dentro de los mercados nacionales e internacionales.
Ve mos tam bién, en el con tex to de los años se sen ta, la po pu larización en norteamerica de los trabajos de T.S. Khun sobre LaEstructuradelas Revoluciones Científicas (1962) que llevó a los intelectuales a percatarse del problema del "cuerpo teórico", planteado por Khun, en algunas ramas de las ciencias sociales, particularmente en la Lingüística y la Economía, que mostraban diferentes variantes dentro del mismo discurso, proble ma que vie ne a re sol ver les Khun, al de no minar, a di chas varian tes, paradigmasovariantes paradigmáticas del mismo discurso.

Lo anterior repercute en las actividades de investigación de los in te lec tua les nor te ame ri ca nos den tro de las áreas men cionadas, quienes se lanzan a la búsqueda de paradigmas,como componentes teóricos de la disciplina, hasta plantear, siguiendo a Khun, los paradigmas como "acuerdos de intelectuales".

\section{CONCLUSIONES}

Si bien es cierto que podemos ha blar de una cier ta in fluen cia de Khun dentro de los problemas que surgieron en el ámbito bi blio te co ló gi co, como en los que se pre sen ta ron en otras disciplinas ya consolidadas como ciencias; no es menos cierto que existía la necesidad real de estructurar teóricamente el campo bibliotecológico, necesidad que alcanza carácter de urgen cia para mu chos biblio te cólogos en la ac tualidad. Necesidad real que debió ha ber se pues to en evi den cia des de la década de los años treinta.

Pro ba ble men te, si se hu biera in sis ti do en la ne ce si dad de formación teórica del bibliotecario, para compensar su formación em pírica, lain ves ti ga ción hu biera te ni do una pre sen ciay un de sarrollo más na tu ral en la edu ca ción y habili da des pos teriores del bibliotecario-bibliotecólogo. La capacidad de investigación hubiera sido la consecuencia obligada de la demanda y realización de una formación también abstracta para com pren der los ni ve les teó ri cos que en esa épo ca ya había alcanzado la bibliotecología.

Em pero, los trein ta años re vi sa dos mues tran, con ra ras ex cepciones, que los bibliotecarios, porsuautoseñaladaformación empírico-téc nica, in sis tie ron en laim por tan cia de los me dios: los mé to dos de in ves ti ga ción, la in ves ti ga ción en sí y la convirtieron en un fin en sí misma.

Será en las si guien tes dé ca das cuan do se bus ca rá en los Es tados Unidos de Norteamérica que la teoría y la investigación encuentren su lugar preciso aunque no sin obstáculos.

\section{NOTAS BIBLIOGRAFICAS}

1.- Williamson, C. “The Place of Research in Library Science” Quarterly J. January 1931 : 1-17

2.- Buckingham, B.R., "A. Challenge to librarians", Journal of Higher Education, Vol. 1 (Febrero 1940), p. 114

3.- En nis, P.N. "Commit ment to Re search: in a Ka lei dos co pic view of li brary re search" in Wil son Library y Bulletin, Col. 41 May. 1967, p. 899. 
4.- Waples, Douglas. Investigating library problems, Chicago, University of Chicago Press, 1939.

5.- Whit worth, T.A. "An Outsi der Looks at Re search in Li bra rianship". "Re search in Li bra rianship". Research in Librarianship. 2 (may. 1969).

6.- Garrinson, Guy. Ed. "Research Methods in Librarianship”. Library trends 13 (July. 1964).

7.- Dym, E. “A New approach. To the de ve lopment of a Te chni cal Thesau rus". Ame ri can Do cu mentation Institute Annual Meeting proceeding 1967.

8.- Luhn, H.P. "A Statistical approach. To Mechanized encoding and Searching of Literature In formation" IBM Journal of Research and Development, 1957.

9.- Shaffer, D.E. The Maturrite of Librarianship As a Profession Metuchen N.J.: the Scarecrow Press, 1968.

10.- Ennis, Philip H. “Commitment to Research"; Wilson Library Bulletin 41 (may. 1967).

11.- Golds tein, Ha rold. "Fah ren heit 902, Li brary Re search Could be Ho tter", Wil son Library Bulle tin 41 (may. 1967).

12.- Shaffer. op. cit.

13.- Khun, T.S.LaEstructuradelasRevoluciones Científicas México, Bre viarios(213)F.C.E. 1975.

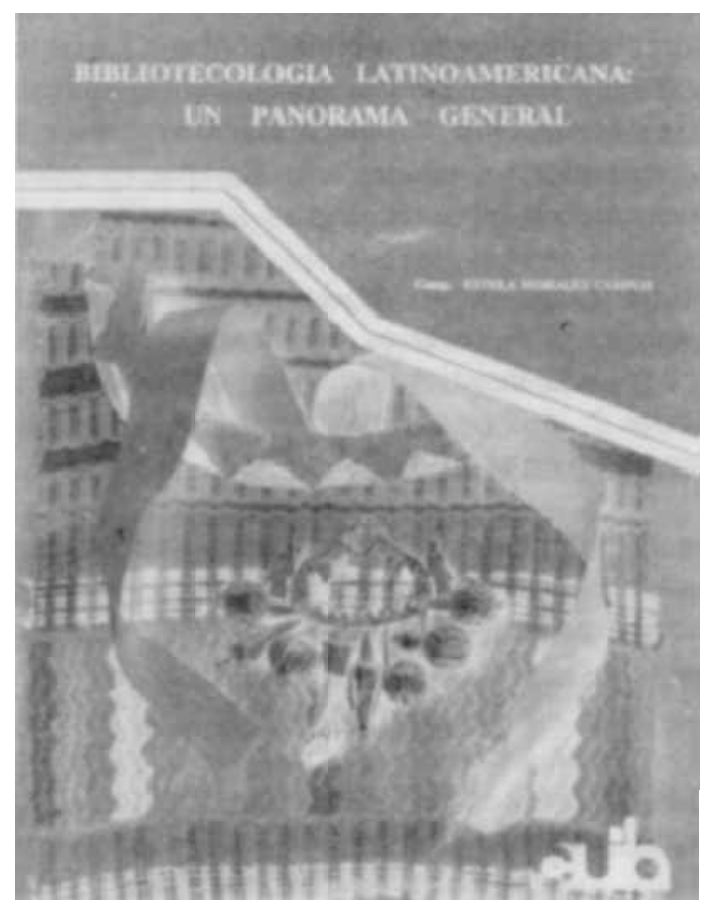

Bibliotecología Latinoamericana: un panorama general. Morales Cam pos, Estela. $164 \mathrm{p}$.

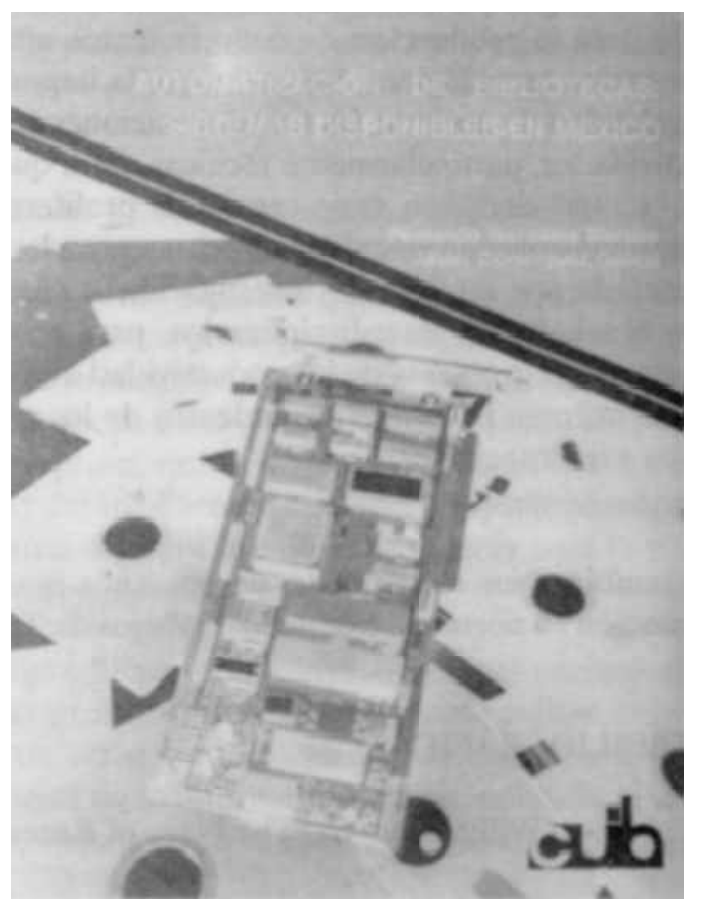

Automatización de bibliotecas: sistemas disponibles en México. González Moreno, Fernando E. y JavierDomínguez G. 140 p. 